\title{
The evaluation of polyglutamine repeats in autosomal dominant Parkinson's
} disease

Chikara Yamashita, $\mathrm{MD}^{1}$; Hiroyuki Tomiyama, MD, $\mathrm{PhD}^{1,2}$; Manabu Funayama, $\mathrm{PhD}^{1,3}$; Saeko Inamizu, $\mathrm{MD}^{4}$; Maya Ando, $\mathrm{MD}^{1}$; Yuanzhe Li, $\mathrm{MD}, \mathrm{PhD}^{3}$; Hiroyo

5 Yoshino, $\mathrm{PhD}^{3}$; Takehisa Araki, $\mathrm{MD}, \mathrm{PhD}^{4}$; Tadashi Ichikawa, $\mathrm{MD}, \mathrm{PhD}^{5}$; Yoshiro Ehara, $\mathrm{PhD}^{6}$; Kinya Ishikawa, $\mathrm{MD}, \mathrm{PhD}^{7}$; Hidehiro Mizusawa, $\mathrm{MD}, \mathrm{PhD}^{7}$; and Nobutaka Hattori ${ }^{*}, \mathrm{MD}, \mathrm{PhD}^{1,2,3}$

101 Department of Neurology, Graduate School of Medicine, Juntendo University, 2-1-1 Hongo, Bunkyo-ku, Tokyo 113-8421, Japan.

2 Department of Neuroscience for Neurodegenerative Disorders, Juntendo University School of Medicine, 2-1-1 Hongo, Bunkyo-ku, Tokyo 113-8421, Japan. 3 Research Institute for Diseases of Old Age, Graduate School of Medicine,

15 Juntendo University, 2-1-1 Hongo, Bunkyo-ku, Tokyo 113-8421, Japan.

4 Hiroshima Red Cross Hospital \& Atomic-bomb Survivors Hospital, 1-9-6

Senda-machi, Naka-ku, Hiroshima 730-8619, Japan.

5 Department of Neurology, Saitama Prefectural Rehablitation Center, 148-1

Nishi-Kaizuka, Ageo-city, Saitama 362-8567, Japan. 
Medicine, 2-1-1 Hongo, Bunkyo-ku, Tokyo 113-8421, Japan.

7 Department of Neurology and Neurological Science, Graduate School, Tokyo Medical and Dental University, 1-5-45 Yushima, Bunkyo-ku, Tokyo 113-8519, Japan.

Word count: abstract: 147 words; text: 1842 words.

Number of references: 36; Figures: 2; Tables: 1; Supplementary Figures: 1;

Supplementary Tables: 3.

\section{Corresponding author:}

Prof. Nobutaka Hattori, MD, PhD,

30 Department of Neurology and Department of Neuroscience for Neurodegenerative

Disorders,

Juntendo University School of Medicine,

2-1-1 Hongo, Bunkyo-ku, Tokyo 113-8421, Japan

Phone: +81-3-5802-1073; Fax: +81-3-5800-0547;

35 E-mail: nhattori@juntendo.ac.jp

\section{Abstract}

We evaluated the contributions of various polyglutamine (polyQ) disease genes to 
Parkinson's disease (PD). We compared the distributions of polyQ repeat lengths in eight common genes (ATXN1, ATXN2, ATXN3, CACNA1A, ATXN7, TBP, ATN1, and $H T T$ ) in 299 unrelated patients with autosomal dominant PD (ADPD) and 329 normal controls. We also analyzed the possibility of genetic interactions between ATXN1 and ATXN2, ATXN2 and ATXN3, and ATXN2 and CACNA1A. Intermediate-length polyQ expansions (>24 Qs) of ATXN2 were found in seven ADPD patients and no controls $(7 / 299=2.34 \%$ and $0 / 329=0 \%$, respectively; $P=0.0053<0.05 / 8$ after Bonferroni correction). These patients showed typical L-DOPA-responsive PD phenotypes.

Conversely, no significant differences in polyQ repeat lengths were found between the ADPD patients and the controls for the other seven genes. Our results may support the hypothesis that $A T X N 2$ polyQ expansion is a specific predisposing factor for multiple

50 neurodegenerative diseases.

Keywords: trinucleotide repeat diseases, Parkinson's disease, polyglutamine,

intermediate length, ataxin-2

\section{Introduction}

Several genes other than the "PARK" genes are suspected to be responsible for parkinsonism. Mutations of these genes sometimes confer symptoms that clinically mimic idiopathic Parkinson's disease (PD) and present radiological or pathological findings characteristic of PD (Klein, et al., 2009). These genes include the 
polyglutamine (polyQ) disease genes: HTT (Walker, 2007), ATXN1 (Dubourg, et al., 1995), ATXN2 (Charles, et al., 2007,Furtado, et al., 2004,Gwinn-Hardy, et al., 2000),

$60 \operatorname{ATXN3}$ (Lu, et al., 2004a,Subramony, et al., 2002), CACNA1A (Kim, et al., 2010), and TBP (Kim, et al., 2009). Of these genes, it has been suggested that intermediate-length polyQ expansions in ATXN2 and TBP are associated with PD (Charles, et al., 2007,Furtado, et al., 2004,Kim, et al., 2009).

In addition, intermediate-length polyQ expansions (24-33 Qs) in ATXN2 have recently been suggested as a risk factor for amyotrophic lateral sclerosis (ALS) (Chen, et al., 2011,Elden, et al., 2010). This observation has inspired several studies investigating how intermediate-length expansions of various polyQ disease genes contribute to neurodegenerative diseases other than those with which they were originally associated (Gispert, et al., 2012,Lee, et al., 2011b,Ross, et al., 2011). Based on these findings and the suggestion that polyQ diseases may share common pathogenic mechanisms(Al-Ramahi, et al., 2007,Bertoni, et al., 2011,Chen and Burgoyne, 2012), we hypothesized that polyQ disease genes in general might play a role in PD. We focused on autosomal dominant PD (ADPD) because polyQ neurodegenerative diseases generally have an AD mode of inheritance, and we compared the distribution of polyQ repeat lengths in eight common genes between 
ADPD patients and normal controls.

\section{Methods}

We conducted genetic analyses of ATXN1, ATXN2, ATXN3, CACNA1A, ATXN7, TBP, ATN1, and HTT in a Japanese cohort with ADPD and normal controls. In this study, we classified the mode of inheritance as autosomal dominant when a family included affected members in two consecutive generations. The diagnosis of PD was confirmed by the participating neurologists based on established criteria (Hughes, et al., 1992).

We recruited the study subjects from the gene bank of our institution. We selected 299 unrelated patients with ADPD (169 females and 130 males; age at onset $(\mathrm{AAO})=57.7$

$85 \pm 13.6(\mathrm{SD})$ years old, range 17-85) from families with unexplained pathogenesis, i.e., those with no known pathogenic mutations in the SNCA, PARK2, LRRK2, and VPS35 genes. A total of 329 healthy unrelated volunteers with no individual or family history of neurodegenerative disease (203 females and 126 males; age at examination $=57.5 \pm$ 11.8(SD) years old, range 23-88) were examined as normal controls. Blood samples

90 were obtained from the patients and controls, all of whom gave informed consent. Our institutional ethics committee approved the genetic study.

DNA was extracted from lymphocytes using standard methods. The polyQ repeat lengths in the polyQ disease genes were detected using capillary electrophoresis with fluorescent 5'-6-FAM-labeled forward primers. The primer sequences and PCR 
conditions are described in Supplementary Table e1. The PCR products were mixed with the LIZ-500 size standard (Applied Biosystems, Foster City, CA) and processed on an Applied Biosystems 3130 Genetic Analyzer (Applied Biosystems) for size determination. The sizes of the repeats were determined with GeneMapper ${ }^{\mathrm{TM}} 3.7$ software (Applied Biosystems).

We evaluated the association between ADPD and the polyQ repeat lengths of each gene using two-tailed Fisher's exact tests, as previously described (Gispert, et al., 2012,Lee, et al., 2011a,Ross, et al., 2011). A $P$ value $<0.05 / 8$ after Bonferroni correction wasconsidered significant ( 8 is for the number of genes investigated in the current study.).

\section{Results}

\subsection{Molecular Genetic Analysis}

The range of repeat lengths in ATXN2 was between 19 and 35. The majority of patients (95.6\% of patients with ADPD and 98.6\% of the controls) had a repeat length

110 of 22, as reported in previous studies (Lee, et al., 2011a,Pulst, et al., 1996). Of the 253 patients with ADPD, 7 harbored repeat lengths longer than 24, whereas none of the controls did (2.8\% and 0\%, respectively; $P=0.0053$, Figure 1 and Table 1$)$. 
No substantial differences in the repeat lengths in ATXN1, ATXN3, CACNA1A,

ATXN7, TBP, ATN1, or HTT were observed between the ADPD patients and controls

115 (Table 1 and Supplementary Figure e1).

We supplementarily sequenced entire coding exons and exon/intron boundaries of glucocerebrosidase gene $(G B A)$ in the 7 probands with intermediate ATXN2 polyQ expansion, because rare $G B A$ mutations have been considered to be a risk factor for PD (Li, et al., 2013,Mitsui, et al., 2009); no GBA mutation was found in these 7 probands.

repeat lengths $>24$.

Figure 2 shows the pedigrees of the seven probands with ATXN2 polyQ repeat lengths

$>24$ and their families. In Family A, AII-2 presented with resting tremor in the

bilateral lower extremities and left-dominant bradykinesia, which were responsive to

L-DOPA and selegiline. AIII-1, who experienced rigidity and resting tremor

predominantly in the left extremities, presented with tongue and jaw tremor

(Supplementary Table e2). All of these signs were relieved by pramipexole. AIII-3 was reportedly initially diagnosed with essential tremor because her first sign was bilateral postural tremor. She underwent left and right thalamotomy at a one-year interval. She

130 showed hyperreflexia in the lower extremities, but this symptom was presumably due 
to cervical spondylosis, for which surgical decompression was performed. AIV-2 and AIV-3, who inherited an intermediate-length polyQ expansion of 35 Qs, were not affected at the time of this study.

In Family B, BI-2 was affected at an older age than her offspring, although their genotypes were the same, and all had L-DOPA-responsive parkinsonism with laterality (Supplementary Table e2).

In Family C, CII-2 was diagnosed with Parkinson's disease with dementia. Although her parents were consanguineous, her polyQ ATXN2 lengths were heterozygous $(29 / 22)$.

140 All other members of the seven families showed L-DOPA-responsive parkinsonism with laterality and were free of motor neuron signs, cerebellar ataxia, and saccadic eye movement disorder. None was reported to have any significant brain magnetic resonance imaging (MRI) abnormality (Supplementary Table e2).

\section{Discussion}

We investigated the distributions of the polyQ repeat lengths of eight common polyQ disease genes (ATXN1, ATXN2, ATXN3, CACNA1A, ATXN7, TBP, ATN1, and HTT) in patients with ADPD. PolyQ repeat lengths > 24 in ATXN2 were significantly more common in the patients than in the controls. To the best of our knowledge, there have 
150 been only two similar studies investigating the distribution of ATXN2 polyQ repeat lengths in PD patients and controls to date (Gispert, et al., 2012,Ross, et al., 2011). Although both previous studies failed to prove any significant difference, one (Gispert, et al., 2012) showed that PD patients tended to have longer repeat lengths, consistent with our results. In the other previous study (Ross, et al., 2011), the controls might have included some number of pre-symptomatic patients because the mean age of the controls was lower than that of the PD patients.

In reference to the recent studies concerning the effect of polyQ repeat length on neurodegenerative disease, we screened for a threshold of the normal ATXN2 polyQ repeat length around a range from 24 to 34 (Charles, et al., 2007,Chen, et al., 2011,Elden, et al., 2010,Gispert, et al., 2012,Lee, et al., 2011a,Lee, et al., 2011b,Ross, et al., 2011). The distribution of our patients differed significantly from that of controls only when the cutoff was set to 25 . This may be much lower than the threshold for ATXN2-related PD adopted by previous studies (Charles, et al., 2007), but it is possible that the cutoff for ATXN2 polyQ repeat length and its influence on PD may vary from population to population, as is the case for ALS, as indicated in a previous study (Lee, et al., 2011b). Such variation of the threshold would be consistent with the observation that previous reports of ATXN2-associated PD have mainly been from East Asian 
populations (Charles, et al., 2007,Klein, et al., 2009,Lu, et al., 2004b,Sun, et al., 2011,Wang, et al., 2009). Additional factors, such as cis- and trans-acting genetic elements, non-allelic genetic modifiers, and stochastic and environmental factors (Charles, et al., 2007,Pulst, et al., 2005), might have enhanced the toxicity of ATXN2 intermediate-length polyQ expansion in our population.

We described the details of family members with ATXN2 intermediate-length expansions (> 24 Qs, Figure 2 and Supplementary Table e2). These patients generally manifested typical PD phenotypes without motor neuron signs, cerebellar ataxia, or saccadic eye movement disorder, as was stated in previous reports (Furtado, et al., 2004,Klein, et al., 2009). A correlation between the association of AAO and polyQ repeat length was not clearly present or absent in our patients with repeat lengths of ATXN2 > 24, as previously observed (Furtado, et al., 2002,Furtado, et al., 2004,Payami, et al., 2003,Sun, et al., 2011). For example, in Family A, members of the $3^{\text {rd }}$ generation had earlier AAOs than did their mother. However, there was a gap between the AAOs of AIII-1 and AIII-3, even though their genotypes were the same. In addition, AIII-1 and AIII-3 had two allele expansions (35/32 Qs) instead of a single allele expansion, which might have caused their early onsets (Ragothaman, et al., 2004). The 35Q alleles may have been inherited 'as is' from AII-1, who reportedly had no neurological 
disorder, although it is also possible that an expansion occurred upon transmission.

Thus, AAOs might be affected by features other than polyQ repeat length, such as genetic and epigenetic factors.

In the current study, we did not find any association between the ADPD phenotype and the repeat lengths of polyQ disease genes other than ATXN2. This result implies that the contribution of ATXN2 to ADPD is due to the specific effects of this gene rather than the presence of the polyQ expansion itself, as reported in a previous study of ALS (Lee, et al., 2011a). This result might appear to be inconsistent with recent reports suggesting that the intermediate polyQ expansion of $T B P$ is likely to be a risk factor for PD (Kim, et al., 2009,Wu, et al., 2004,Xu, et al., 2010, Yun, et al., 2011). However, because those reports did not provide significant evidence, and because all of these studies were performed in East Asian patients, further evidence should be accumulated.

As a supplementary analysis, we also applied a multiple logistic regression including the product terms $A T X N 1 * A T X N 2, A T X N 2 * A T X N 3$, and ATXN2*CACNA1A in order to screen for some interactions among these polyQ disease gene combinations, based on previous studies showing the possibility of interaction among these polyQ genes (Al-Ramahi, et al., 2007,Jardim, et al., 2003,Lessing and Bonini, 2008,Pulst, et al., 
2005). However, no significant difference was detected between the PD patients and

In conclusion, an intermediate-length polyQ expansion of ATXN2 is likely to

contribute to the pathogenesis of ADPD, either directly causing the PD phenotype or modifying the effects of unknown genes on the PD phenotype. Our results add to the recent finding that intermediate-length polyQ repeat expansions of ATXN2 may be a

210 contributing factor in multiple neurodegenerative diseases.

\section{Acknowledgments}

The authors thank all the participants in this study. 
Yamashita $\mathrm{C}$ et al., Page 13 


\section{References}

Al-Ramahi, I., Perez, A.M., Lim, J., Zhang, M., Sorensen, R., de Haro, M., Branco, J., Pulst, S.M., Zoghbi, H.Y., Botas, J. 2007. dAtaxin-2 mediates expanded Ataxin-1-induced neurodegeneration in a Drosophila model of SCA1. PLoS Genet 3(12), e234. doi:07-PLGE-RA-0498 [pii]

10.1371/journal.pgen.0030234.

Bertoni, A., Giuliano, P., Galgani, M., Rotoli, D., Ulianich, L., Adornetto, A., Santillo, M.R., Porcellini, A., Avvedimento, V.E. 2011. Early and late events induced by polyQ-expanded proteins: identification of a common pathogenic property of polYQ-expanded proteins. J Biol Chem 286(6), 4727-41. doi:M110.156521 [pii]

10.1074/jbc.M110.156521.

Charles, P., Camuzat, A., Benammar, N., Sellal, F., Destee, A., Bonnet, A.M., Lesage, S., Le Ber, I., Stevanin, G., Durr, A., Brice, A. 2007. Are interrupted SCA2 CAG repeat expansions responsible for parkinsonism? Neurology 69(21), 1970-5. doi:01.wnl.0000269323.21969.db [pii]

10.1212/01.wnl.0000269323.21969.db.

Chen, X., Burgoyne, R.D. 2012. Identification of common genetic modifiers of neurodegenerative diseases from an integrative analysis of diverse genetic screens in model organisms. BMC Genomics 13, 71. doi:1471-2164-13-71 [pii] 10.1186/1471-2164-13-71.

Chen, Y., Huang, R., Yang, Y., Chen, K., Song, W., Pan, P., Li, J., Shang, H.F. 2011. Ataxin-2 intermediate-length polyglutamine: a possible risk factor for Chinese patients with amyotrophic lateral sclerosis. Neurobiol Aging 32(10), 1925 e1-5. doi:S0197-4580(11)00198-9 [pii]

10.1016/j.neurobiolaging.2011.05.015.

Dubourg, O., Durr, A., Cancel, G., Stevanin, G., Chneiweiss, H., Penet, C., Agid, Y., Brice, A. 1995. Analysis of the SCA1 CAG repeat in a large number of families with dominant ataxia: clinical and molecular correlations. Ann Neurol 37(2), 176-80. doi:10.1002/ana.410370207.

Elden, A.C., Kim, H.J., Hart, M.P., Chen-Plotkin, A.S., Johnson, B.S., Fang, X., Armakola, M., Geser, F., Greene, R., Lu, M.M., Padmanabhan, A., Clay-Falcone, D., McCluskey, L., Elman, L., Juhr, D., Gruber, P.J., Rub, U., Auburger, G., Trojanowski, J.Q., Lee, V.M., Van Deerlin, V.M., Bonini, N.M., Gitler, A.D. 2010. Ataxin-2 intermediate-length polyglutamine expansions are associated with increased risk for ALS. Nature 466(7310), 1069-75. 
doi:nature09320 [pii]

10.1038/nature09320.

Furtado, S., Farrer, M., Tsuboi, Y., Klimek, M.L., de la Fuente-Fernandez, R., Hussey, J., Lockhart, P., Calne, D.B., Suchowersky, O., Stoessl, A.J., Wszolek, Z.K. 2002. SCA-2 presenting as parkinsonism in an Alberta family: clinical, genetic, and PET findings. Neurology 59(10), 1625-7.

Furtado, S., Payami, H., Lockhart, P.J., Hanson, M., Nutt, J.G., Singleton, A.A., Singleton, A., Bower, J., Utti, R.J., Bird, T.D., de la Fuente-Fernandez, R., Tsuboi, Y., Klimek, M.L., Suchowersky, O., Hardy, J., Calne, D.B., Wszolek, Z.K., Farrer, M., Gwinn-Hardy, K., Stoessl, A.J. 2004. Profile of families with parkinsonism-predominant spinocerebellar ataxia type 2 (SCA2). Mov Disord 19(6), 622-9. doi:10.1002/mds.20074.

275 Gispert, S., Kurz, A., Waibel, S., Bauer, P., Liepelt, I., Geisen, C., Gitler, A.D., Becker, T., Weber, M., Berg, D., Andersen, P.M., Kruger, R., Riess, O., Ludolph, A.C., Auburger, G. 2012. The modulation of Amyotrophic Lateral Sclerosis risk by ataxin-2 intermediate polyglutamine expansions is a specific effect. Neurobiol Dis 45(1), 356-61. doi:S0969-9961(11)00287-7 [pii]

$280 \quad$ 10.1016/j.nbd.2011.08.021.

Gwinn-Hardy, K., Chen, J.Y., Liu, H.C., Liu, T.Y., Boss, M., Seltzer, W., Adam, A., Singleton, A., Koroshetz, W., Waters, C., Hardy, J., Farrer, M. 2000. Spinocerebellar ataxia type 2 with parkinsonism in ethnic Chinese. Neurology 55(6), 800-5.

285 Hands, S., Sinadinos, C., Wyttenbach, A. 2008. Polyglutamine gene function and dysfunction in the ageing brain. Biochim Biophys Acta 1779(8), 507-21. doi:S1874-9399(08)00119-3 [pii]

10.1016/j.bbagrm.2008.05.008.

Hughes, A.J., Daniel, S.E., Kilford, L., Lees, A.J. 1992. Accuracy of clinical diagnosis of idiopathic Parkinson's disease: a clinico-pathological study of 100 cases. J Neurol Neurosurg Psychiatry 55(3), 181-4.

Jardim, L., Silveira, I., Pereira, M.L., do Ceu Moreira, M., Mendonca, P., Sequeiros, J., Giugliani, R. 2003. Searching for modulating effects of SCA2, SCA6 and DRPLA CAG tracts on the Machado-Joseph disease (SCA3) phenotype. Acta Neurol Scand 107(3), 211-4. doi:046 [pii].

Kim, J.M., Lee, J.Y., Kim, H.J., Kim, J.S., Kim, Y.K., Park, S.S., Kim, S.E., Jeon, B.S. 2010. The wide clinical spectrum and nigrostriatal dopaminergic damage in spinocerebellar ataxia type 6. J Neurol Neurosurg Psychiatry 81(5), 529-32. 
doi:81/5/529 [pii]

10.1136/jnnp.2008.166728.

Kim, J.Y., Kim, S.Y., Kim, J.M., Kim, Y.K., Yoon, K.Y., Lee, B.C., Kim, J.S., Paek, S.H., Park, S.S., Kim, S.E., Jeon, B.S. 2009. Spinocerebellar ataxia type 17 mutation as a causative and susceptibility gene in parkinsonism. Neurology 72(16), 1385-9. doi:72/16/1385 [pii]

Klein, C., Schneider, S.A., Lang, A.E. 2009. Hereditary parkinsonism: Parkinson disease look-alikes--an algorithm for clinicians to "PARK" genes and beyond. Mov Disord 24(14), 2042-58. doi:10.1002/mds.22675.

Lee, T., Li, Y.R., Chesi, A., Hart, M.P., Ramos, D., Jethava, N., Hosangadi, D., Epstein, J., Hodges, B., Bonini, N.M., Gitler, A.D. 2011a. Evaluating the prevalence of polyglutamine repeat expansions in amyotrophic lateral sclerosis. Neurology 76(24), 2062-5. doi:WNL.0b013e31821f4447 [pii]

10.1212/WNL.0b013e31821f4447.

Lee, T., Li, Y.R., Ingre, C., Weber, M., Grehl, T., Gredal, O., de Carvalho, M., Meyer, T., Tysnes, O.B., Auburger, G., Gispert, S., Bonini, N.M., Andersen, P.M., Gitler, A.D. 2011b. Ataxin-2 intermediate-length polyglutamine expansions in European ALS patients. Hum Mol Genet 20(9), 1697-700. doi:ddr045 [pii] 10.1093/hmg/ddr045.

Lessing, D., Bonini, N.M. 2008. Polyglutamine genes interact to modulate the severity and progression of neurodegeneration in Drosophila. PLoS Biol 6(2), e29. doi:07-PLBI-RA-3100 [pii]

10.1371/journal.pbio.0060029.

Li, Y., Sekine, T., Funayama, M., Li, L., Yoshino, H., Nishioka, K., Tomiyama, H., Hattori, N. 2013. Clinicogenetic study of GBA mutations in patients with familial Parkinson's disease. Neurobiology of aging. doi:10.1016/j.neurobiolaging.2013.09.019.

Lu, C.S., Chang, H.C., Kuo, P.C., Liu, Y.L., Wu, W.S., Weng, Y.H., Yen, T.C., Chou, Y.H. 2004a. The parkinsonian phenotype of spinocerebellar ataxia type 3 in a Taiwanese family. Parkinsonism Relat Disord 10(6), 369-73. doi:10.1016/j.parkreldis.2004.03.009

S1353802004000690 [pii].

Lu, C.S., Wu Chou, Y.H., Kuo, P.C., Chang, H.C., Weng, Y.H. 2004b. The parkinsonian phenotype of spinocerebellar ataxia type 2. Arch Neurol 61(1), 35-8. doi:10.1001/archneur.61.1.35 
61/1/35 [pii].

Mitsui, J., Mizuta, I., Toyoda, A., Ashida, R., Takahashi, Y., Goto, J., Fukuda, Y., Date, H., Iwata, A., Yamamoto, M., Hattori, N., Murata, M., Toda, T., Tsuji, S. 2009. Mutations for Gaucher disease confer high susceptibility to Parkinson disease. Archives of neurology 66(5), 571-6. doi:10.1001/archneurol.2009.72.

Payami, H., Nutt, J., Gancher, S., Bird, T., McNeal, M.G., Seltzer, W.K., Hussey, J., Lockhart, P., Gwinn-Hardy, K., Singleton, A.A., Singleton, A.B., Hardy, J., Farrer, M. 2003. SCA2 may present as levodopa-responsive parkinsonism. Mov Disord 18(4), 425-9. doi:10.1002/mds.10375.

Pulst, S.M., Nechiporuk, A., Nechiporuk, T., Gispert, S., Chen, X.N., Lopes-Cendes, I.,

Pearlman, S., Starkman, S., Orozco-Diaz, G., Lunkes, A., DeJong, P., Rouleau, G.A., Auburger, G., Korenberg, J.R., Figueroa, C., Sahba, S. 1996. Moderate expansion of a normally biallelic trinucleotide repeat in spinocerebellar ataxia type 2. Nat Genet 14(3), 269-76. doi:10.1038/ng1196-269.

Pulst, S.M., Santos, N., Wang, D., Yang, H., Huynh, D., Velazquez, L., Figueroa, K.P. 2005. Spinocerebellar ataxia type 2: polyQ repeat variation in the CACNA1A calcium channel modifies age of onset. Brain 128( $\mathrm{Pt} 10), 2297-303$. doi:awh586 [pii]

10.1093/brain/awh586.

Ragothaman, M., Sarangmath, N., Chaudhary, S., Khare, V., Mittal, U., Sharma, S., Komatireddy, S., Chakrabarti, S., Mukerji, M., Juyal, R.C., Thelma, B.K., Muthane, U.B. 2004. Complex phenotypes in an Indian family with homozygous SCA2 mutations. Ann Neurol 55(1), 130-3. doi:10.1002/ana.10815.

Ross, O.A., Rutherford, N.J., Baker, M., Soto-Ortolaza, A.I., Carrasquillo, M.M., DeJesus-Hernandez, M., Adamson, J., Li, M., Volkening, K., Finger, E., Seeley, W.W., Hatanpaa, K.J., Lomen-Hoerth, C., Kertesz, A., Bigio, E.H., Lippa, C., Woodruff, B.K., Knopman, D.S., White, C.L., 3rd, Van Gerpen, J.A., Meschia, J.F., Mackenzie, I.R., Boylan, K., Boeve, B.F., Miller, B.L., Strong, M.J., Uitti, R.J., Younkin, S.G., Graff-Radford, N.R., Petersen, R.C., Wszolek, Z.K., Dickson, D.W., Rademakers, R. 2011. Ataxin-2 repeat-length variation and neurodegeneration. Hum Mol Genet 20(16), 3207-12. doi:ddr227 [pii]

10.1093/hmg/ddr227.

Sequeiros, J., Seneca, S., Martindale, J. 2010. Consensus and controversies in best practices for molecular genetic testing of spinocerebellar ataxias. Eur J Hum Genet 18(11), 1188-95. doi:ejhg201010 [pii] 
10.1038/ejhg.2010.10.

Subramony, S.H., Hernandez, D., Adam, A., Smith-Jefferson, S., Hussey, J., Gwinn-Hardy, K., Lynch, T., McDaniel, O., Hardy, J., Farrer, M., Singleton, A. 2002. Ethnic differences in the expression of neurodegenerative disease: Machado-Joseph disease in Africans and Caucasians. Mov Disord 17(5), 1068-71. doi:10.1002/mds.10241.

Sun, H., Satake, W., Zhang, C., Nagai, Y., Tian, Y., Fu, S., Yu, J., Qian, Y., Chu, J., Toda, T. 2011. Genetic and clinical analysis in a Chinese parkinsonism-predominant spinocerebellar ataxia type 2 family. J Hum Genet 56(4), 330-4. doi:jhg201114 [pii]

10.1038/jhg.2011.14.

Walker, F.O. 2007. Huntington's disease. Lancet 369(9557), 218-28. doi:S0140-6736(07)60111-1 [pii]

10.1016/S0140-6736(07)60111-1.

385 Wang, J.L., Xiao, B., Cui, X.X., Guo, J.F., Lei, L.F., Song, X.W., Shen, L., Jiang, H., Yan, X.X., Pan, Q., Long, Z.G., Xia, K., Tang, B.S. 2009. Analysis of SCA2 and SCA3/MJD repeats in Parkinson's disease in mainland China: genetic, clinical, and positron emission tomography findings. Mov Disord 24(13), 2007-11. doi:10.1002/mds.22727.

Wu, Y.R., Lin, H.Y., Chen, C.M., Gwinn-Hardy, K., Ro, L.S., Wang, Y.C., Li, S.H., Hwang, J.C., Fang, K., Hsieh-Li, H.M., Li, M.L., Tung, L.C., Su, M.T., Lu, K.T., Lee-Chen, G.J. 2004. Genetic testing in spinocerebellar ataxia in Taiwan: expansions of trinucleotide repeats in SCA8 and SCA17 are associated with typical Parkinson's disease. Clin Genet 65(3), 209-14. doi:213 [pii].

Xu, Q., Jia, D., Wang, J., Guo, J., Jiang, H., Lei, L., Shen, L., Pan, Q., Xia, K., Yan, X., Tang, B. 2010. Genetic analysis of Spinocerebellar ataxia type 17 in Parkinson's disease in Mainland China. Parkinsonism Relat Disord 16(10), 700-2. doi:S1353-8020(10)00218-X [pii]

10.1016/j.parkreldis.2010.08.020.

400 Yun, J.Y., Lee, W.W., Kim, H.J., Kim, J.S., Kim, J.M., Kim, S.Y., Kim, J.Y., Park, S.S., Kim, Y.K., Kim, S.E., Jeon, B.S. 2011. Relative contribution of SCA2, SCA3 and SCA17 in Korean patients with parkinsonism and ataxia. Parkinsonism Relat Disord 17(5), 338-42. doi:S1353-8020(11)00020-4 [pii]

10.1016/j.parkreldis.2011.01.015. 
Figure Legends

Figure 1

The distribution of polyglutamine (polyQ) repeat lengths of $A T X N 2$ in autosomal

410 dominant Parkinson's disease patients and normal controls.

The histogram shows only subjects with $\geq 23$ repeats.

\section{Figure 2}

The pedigrees of 7 families in which the proband has an $A T X N 2$ polyQ repeat

415 length $>\mathbf{2 4}$.

ATXN2 repeat lengths are listed above and to the right of the pedigree symbols of the genotyped individuals. 
Table 1.

420 Fisher's exact tests of polyQ repeat lengths between ADPD patients and controls.

\begin{tabular}{cccc}
$\begin{array}{c}\text { PolyQ } \\
\text { disease } \\
\text { gene }\end{array}$ & PolyQ repeat length & $\begin{array}{c}\text { Conventional } \\
\text { normal } \\
\text { range } \dagger\end{array}$ & $\begin{array}{c}\text { Difference between } \\
\text { ADPD patients and } \\
\text { controls? }\end{array}$ \\
\hline ATXN1 & $21-36$ & $6-44$ & No \\
ATXN2 & $\begin{array}{c}19-35 \\
\text { 25-35Qs: } 2.3 \% \text { of ADPD, 0\% } \\
\text { of control }\end{array}$ & $14-31$ & $\begin{array}{c}\text { Yes, } P=0.0053 \\
(<0.05 / 8), \text { OR }=\infty\end{array}$ \\
ATXN3 & $13-46$ & $11-44$ & No \\
CACNA1A & $5-18$ & $4-18$ & No \\
ATXN7 & $1-10$ & $4-19$ & No \\
TBP & $30-40$ & $25-42$ & No \\
ATN1 & $12-36$ & $6-35$ & No \\
HTT & $15-35$ & $6-34$ & No \\
\hline
\end{tabular}

ADPD: autosomal dominant Parkinson's disease; Q: glutamine.

$\dagger$ The consensus normal ranges of the polyQ repeat lengths associated with the corresponding disease (e.g., ATXN1 for SCA1). (Hands, et al., 2008,Sequeiros, et al., 2010) 
Figure 1

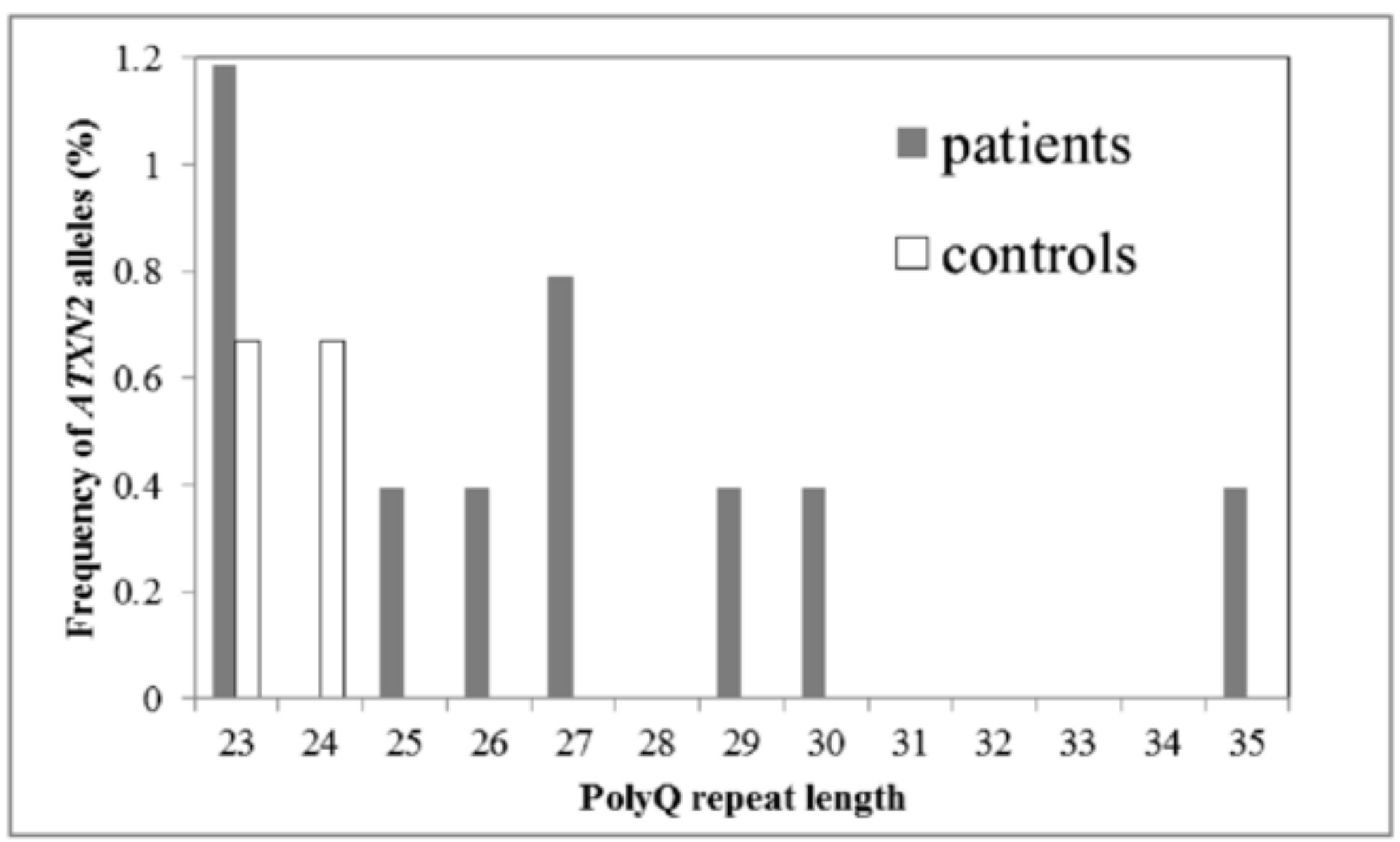

\begin{tabular}{c|ccccccccccccc} 
PolyQ length & 19 & $20-21$ & 22 & 23 & 24 & 25 & 26 & 27 & 28 & 29 & 30 & $31-34$ & 35 \\
\hline Patients & 1 & 0 & 288 & 3 & 0 & 1 & 1 & 2 & 0 & 1 & 1 & 0 & 1 \\
Controls & 0 & 0 & 325 & 2 & 2 & 0 & 0 & 0 & 0 & 0 & 0 & 0 & 0
\end{tabular}




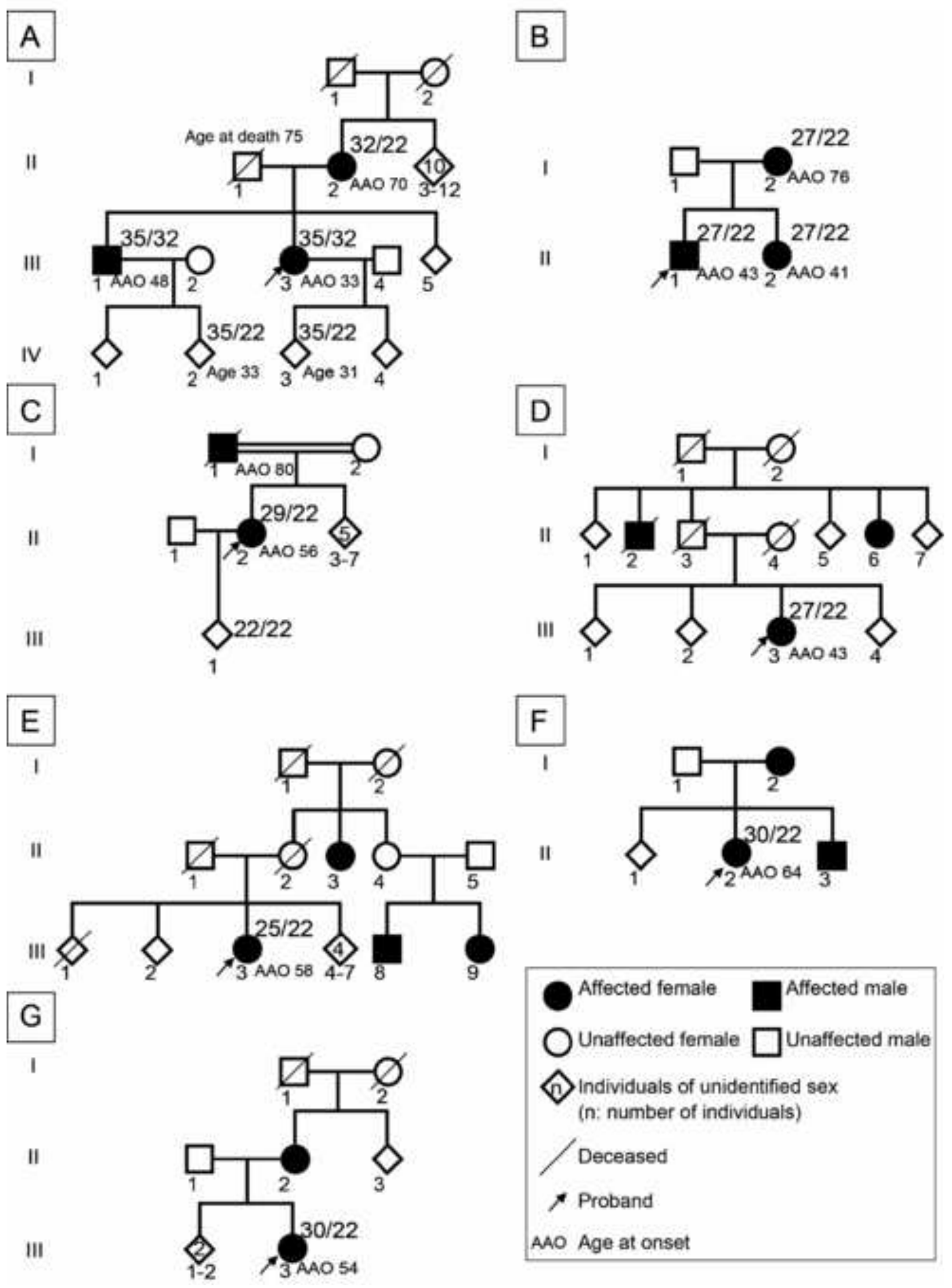

